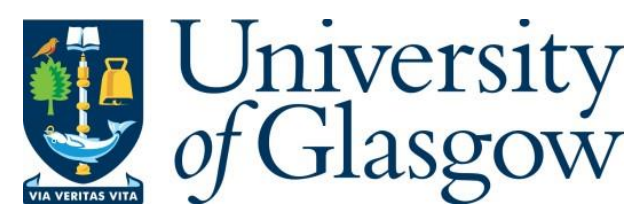

Liang, J., Lee, J., Berrada, S., Georgiev, V., Pandey, R. R., Chen, R., Asenov, A. and Todri-Sanial, A. (2018) Atomistic to circuit-level modeling of doped SWCNT for onchip interconnects. IEEE Transactions on Nanotechnology, (doi:10.1109/TNANO.2018.2802320).

There may be differences between this version and the published version. You are advised to consult the publisher's version if you wish to cite from it.

$\underline{\text { http://eprints.gla.ac.uk/157009/ }}$

Deposited on: 9 February 2018

Enlighten - Research publications by members of the University of Glasgow http://eprints.gla.ac.uk 


\title{
Atomistic- to Circuit-Level Modeling of Doped SWCNT for On-Chip Interconnects
}

\author{
Jie Liang, Jaehyun Lee, Salim Berrada, Vihar P. Georgiev, Member, IEEE, Reeturaj Pandey, \\ Rongmei Chen, Member, IEEE, Asen Asenov, Fellow, IEEE and Aida Todri-Sanial, Member, IEEE
}

\begin{abstract}
In this article, we present a hierarchical model for doped single-walled carbon nanotube (SWCNT) for onchip interconnect application. Our model aims to study CVD grown SWCNTs while considering defects and contacts to metal electrodes. Both defects and poor contacts can worsen CNT conductivities and ultimately deteriorate their interconnect performance. We investigate the fundamental physical mechanism of charge based doping with the purpose of improving SWCNT electrical conductivity as well as a potential solution to alleviating the impact of defects and contact resistances. We present an atomistic model to study the number of conducting channels of doped SWCNT with different vacancy defect configurations. Circuit-level electrical modeling and simulations are performed on SWCNT interconnect while considering the impact of doping, defects and contact resistance. Simulation results show up to $80 \%$ resistance reduction by doping where $17 \%$ of delay increases due to defects. Additionally, we observe doping can mitigate the impact of defects by more than $12 \%$, but there is almost no improvement in the contact resistance.
\end{abstract}

Index Terms-carbon nanotubes, defective SWCNT, doped SWCNT, on-chip interconnect.

\section{INTRODUCTION}

W ITH technology node scaling down, the performance of on-chip interconnects is degrading due to increased parasitics and becoming even more critical for ultra large-scale integrated (ULSI) circuit performance. Conventional copper $(\mathrm{Cu})$ interconnects have started to reach their limits by the continuous shrinking of dimensions and high aspect ratio requirements [1], [2]. The large current flows and elevated on-chip temperatures introduce severe electromigration issues which are worsening with sub $10 \mathrm{~nm}$ scaling [3]. Finding a new material to replace $\mathrm{Cu}$ as on-chip back-end-of-line interconnect material has attracted a lot of research interest and is an on-going quest [4], [5]. Carbon nanotubes (CNTs) due to their ballistic transport and high thermal conductivity are considered as a potential candidate for future on-chip interconnects [6]. Nevertheless, controlling the chirality of CNTs is still not trivial and under many investigations [7][9].

This work was supported by the European Commission H2020 CONNECT project under grant agreement number 688612. (http://www.connecth2020.eu/).

J. Liang, R. Pandey, R. Chen and A. Todri-Sanial are with the Microelectronics Department, CNRS-LIRMM/University of Montpellier, Montpellier, 34095 France. E-mails: jie.liang@lirmm.fr and aida.todri@lirmm.fr.

J. Lee, S. Berrada, V. P. Georgiev, and A. Asenov are with the School of Engineering, University of Glasgow, Glasgow, G12 8QQ UK.

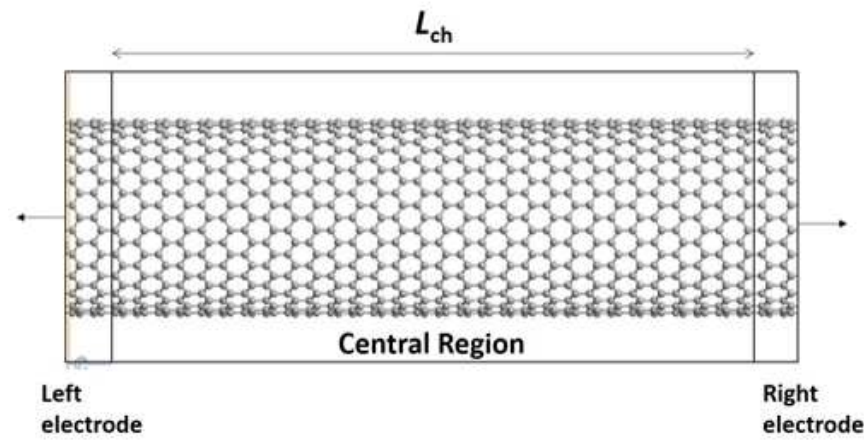

Fig. 1. Atomistic simulation geometry of $\operatorname{SWCNT}(24,0)$ for interconnect configuration. Two semi-infinite electrodes, and length of defective region $L_{c h}$ is set as $42.6 \mathrm{~nm}$.

In this work, we investigate charge-based doping of CNTs as a means to alleviate the impact of random chirality distribution. Based on the atomistic simulation, it was shown that doping degenerates semiconducting to metallic CNTs which are suitable for interconnect application [10]. We further investigate doping on CNTs to understand its impact on CNT conductance and performance. Additionally, we study defects which might be introduced during the CNT growth process [11] and consequently can impact the CNT performance and power dissipation for on-chip interconnects. Moreover, the metal-CNT contact resistance is also an essential factor for fast, and reliable interconnect application. In this paper, we investigate doped, and defective SWCNT including contact resistances for back-end-of-line (BEOL) on-chip interconnects.

\section{HierarchicAl MODEL}

\section{A. Atomistic modeling}

Atomistix ToolKit (ATK) [12] implemented by the framework of tight-binding (TB) Non-Equilibrium Greens Function (NEGF) has been used for atomistic modeling. An extended TB approach, which includes the third nearest neighbor hopping is used for describing the CNTs Hamiltonian [13]. We use the ballistic approximation for all transport calculations.

Figure 1 shows the geometry of metallic zigzag SWCNT $(24,0)$ for an interconnect configuration. Left and right electrodes are semi-infinite electrodes with the defective region (central region) in between. We only consider vacancy type of defects where several carbon atoms can be missing from the SWCNT. In the central region, we distribute vacancy defects 
TABLE I

STATISTICAL VALUES OF DEFECTIVE RESISTANCE FOR SWCNT $(24,0)$

\begin{tabular}{cccc}
\hline $\begin{array}{c}\text { Length of SWCNT } \\
\text { defective region }(\mathrm{nm})\end{array}$ & $\begin{array}{c}\text { SWCNT } \\
\text { Diameter (Ang) }\end{array}$ & $\begin{array}{c}\text { Number of } \\
\text { defects }\end{array}$ & $\begin{array}{c}\text { Defective } \\
\text { resistance }(\mathrm{k} \Omega)\end{array}$ \\
\hline \hline $42.6(100 \mathrm{UC})$ & 18.8 & 1 & 4.028 \\
\hline $42.6(100 \mathrm{UC})$ & 18.8 & 2 & 12.469 \\
\hline $42.6(100 \mathrm{UC})$ & 18.8 & 3 & 15.241 \\
\hline $42.6(100 \mathrm{UC})$ & 18.8 & 4 & 26.380 \\
\hline $42.6(100 \mathrm{UC})$ & 18.8 & 5 & 31.268 \\
\hline $42.6(100 \mathrm{UC})$ & 18.8 & 6 & 39.499 \\
\hline
\end{tabular}

randomly from 1 to 6 . We compute the defective resistance as $R_{\text {defect }}=R_{\text {total }}-R_{\text {ballistic }}$ where $R_{\text {total }}$ is calculated by TBNEGF. Please note that the length of the defective region does not impact the defective SWCNT resistance as was shown in [14]. The length of the defective region is set to $42.6 \mathrm{~nm}$ and we observe that distribution of defective resistance varies slightly with the length, however the defective resistance mean values remain almost unchanged. This is because the interaction between defects is shorter than their phase relaxation length in our atomistic configuration.

Fig. 2 shows a statistical analysis and derivation of the median value of defective resistance for each configuration. 80 samples were used for the calculation. Specific values are shown in Table 1. Defective resistance mean value varies from 4 to $40 \mathrm{k} \Omega$ with 1 to 6 defects, respectively. In [14], it was shown a small variation of defective resistance mean value for both metallic zigzag and armchair SWCNT. Our models and simulation approach are suitable for both zigzag and armchair type of SWCNTs.

Density Functional Theory (DFT) with Generalized Gradient Approximation (GGA) is used to calculate the conductance $G$ of SWCNT with respect to the different position of Fermi energy level. We assume a) there are no additional states due to dopant; b) there is no change of Density of State (DOS) or energy band structure due to dopant. The number of conducting channels $N_{C}$ is derived by $G / G_{0}$, where $G_{0}$ is the quantum conductance $2 e^{2} / \mathrm{h}\left(G_{0}=7.74810^{-5} \mathrm{~S}\right)$. Fig. 3 shows the results of DFTGGA calculation for deriving the number of conducting channels as a function of Fermi-level energy.

\section{B. Electrical compact modeling}

Electrical models of CNT for interconnect application have already been developed and widely used by our community. Most notably, [15] and [16] have investigated modeling and simulation of pristine single-walled (SW) and multi-walled (MW) CNTs for on-chip interconnects. Based on their work, we explore here the number of conducting channels $N_{C}$ on CNTs as an important physical parameter for doped CNTs. We also take into account the resistance due to defects. We develop a hierarchical model and simulation method to assess the performance of fabricated SWCNTs (i.e., defects, impurities, contacts) realistically for on-chip interconnect applications.

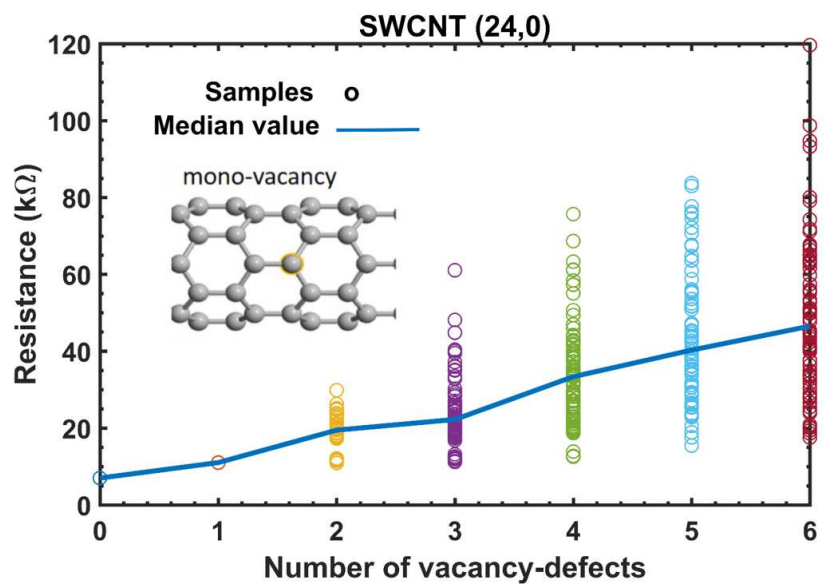

Fig. 2. Dependence of resistance on the number of vacancy defects for a pristine SWCNT $(24,0)$ with 80 samples used for statistical results.

Controlling of the chirality and variability of CVD grown CNTs (i.e., variations on diameter, contacts, number of shells, defects, etc.) remains a challenge. In this paper, we investigate doping as a way to enhance metallic CNTs behavior while alleviating the impact of defects and poor contacts. Large doping concentrations introduce more conducting channels for CNTs [17]. From semiconductor physics and atomistic simulations, we understand that doping shifts the Fermilevel of SWCNT. Moreover, we know from quantum physics that the electron transport (i.e., conducting property) occurs around the Fermi-level. Hence, the Fermi-level shifting by doping introduces more electron subbands available for charge transport on SWCNTs with respect to the particular FermiDirac energy band. From Fig. 3, a pristine metallic SWCNT has two conducting channels (at $0 \mathrm{eV}$ ) and after shifting the Fermi-level position to $1 \mathrm{eV}$ (due to doping), up to 8 conducting channels are introduced.

We also consider defects that occur during the CNT growth process. Defects on SWCNT are modeled as a defective resistance. Based on the atomistic simulation results, we incorporate the defective resistance into our compact SWCNT electrical model. We also investigate the contact resistance on SWCNTs. Contact resistance plays a critical role in interconnect performance and reliability. By taking into account, the impact of doping, defects and contact resistance, our compact model for SWCNT electrical resistance is as:

$$
R_{S W C N T}=\frac{h}{2 e^{2} N_{C}}\left(1+\frac{L}{L_{m f p}}\right)+R_{\text {defect }}+R_{\text {contact }}
$$

where $L_{m f p}$ is the electron mean free path [18]. We model the SWCNT interconnect as a RC-model where parasitic resistance $\mathrm{R}$ is given by equation (1), and parasitic capacitance $\mathrm{C}$ is modeled as quantum capacitance $C_{Q}$ connected in series with electrostatic capacitance $C_{E}$, as shown in equation (2), similar to [15]. Doped $C_{Q}$ is linearly dependent on $N_{C}$. If $N_{C}$ gets larger, $C_{E}$ will have the tendency to dominate $C_{S W C N T}$.

$$
C_{S W C N T}=\frac{C_{Q} C_{E}}{C_{Q}+C_{E}}
$$




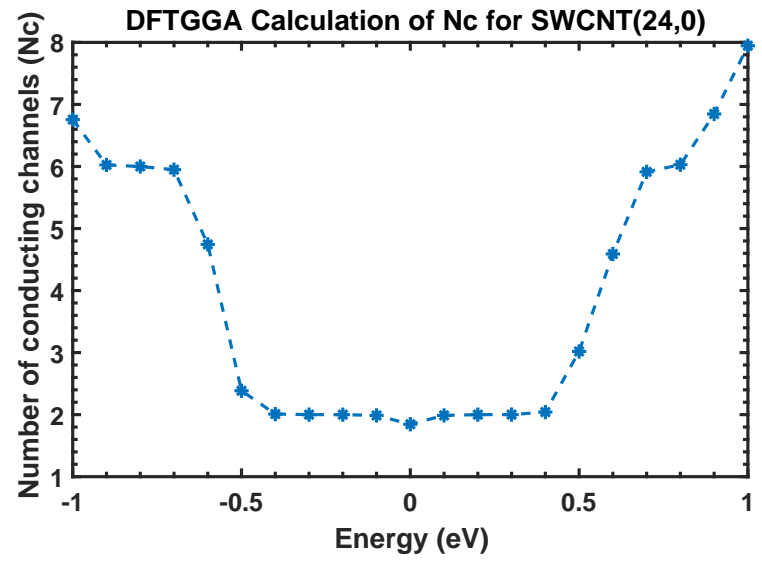

Fig. 3. DFTGGA calculation of number of conducting channels $N c$ for SWCNT $(24,0)$ over different Fermi-level energy positions.

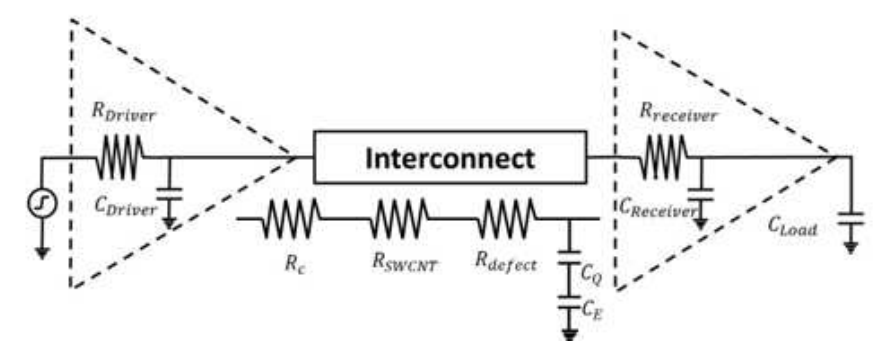

Fig. 4. Circuit level "driver-interconnect-receiver" benchmark for delay simulation.

To evaluate the performance of a defective doped SWCNT with contact resistance, we use a circuit benchmark as shown in Fig. 4. Specific values of $R_{d r i v e r}, C_{d r i v e r}$ and $C_{\text {load }}$ used in our simulations are $24 \mathrm{k} \Omega, 450 \mathrm{aF}$ and $10 \mathrm{fF}$ respectively, which are values extracted from the logic gates implemented in CMOS $45 \mathrm{~nm}$ technology node.

\section{Simulation Results}

We perform our circuit simulations using the Cadence environment with Verilog-A models to represent the SWCNT interconnects between logic gates. We vary the number of SWCNT conducting channels from 2 to 8 to represent different doping concentrations. We also study vacancy-type defects by varying SWCNT resistance as given in Table 1. Additionally, we include the contact resistance varying from 0 to $10 \mathrm{k} \Omega$ to represent side contact with different contacting lengths [19].

Figure 5 shows the pristine SWCNT resistance varying with length and diameter. There are no defects and contact resistance. We note that the resistance increases dramatically with lengths longer than $1 \mu \mathrm{m}$. From theoretical understanding, we know that the mean free path of a SWCNT with $D=1 \mathrm{~nm}$ is about $1 \mu \mathrm{m}$; above this length, the scattering resistance starts to impact the total SWCNT resistance. We also see that resistance decreases for larger diameter SWCNTs as larger diameters provide more metallic conducting property.

Figure 6 shows the resistance of doped SWCNT $(24,0)$ with $D=18.8$ Ang as a function of length and number of conducting channels $N_{C}$. With $N_{C}$ increasing to 8 , the

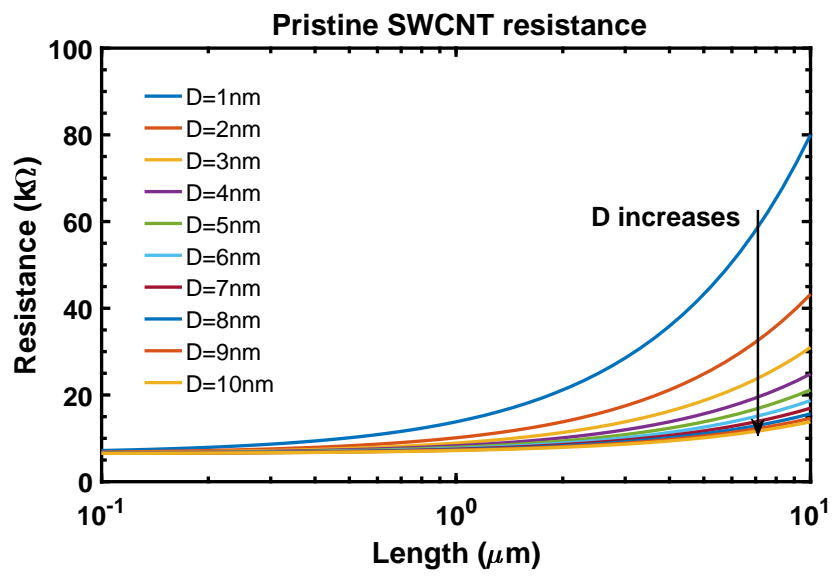

Fig. 5. Resistance of pristine SWCNT varying with length for different diameters.

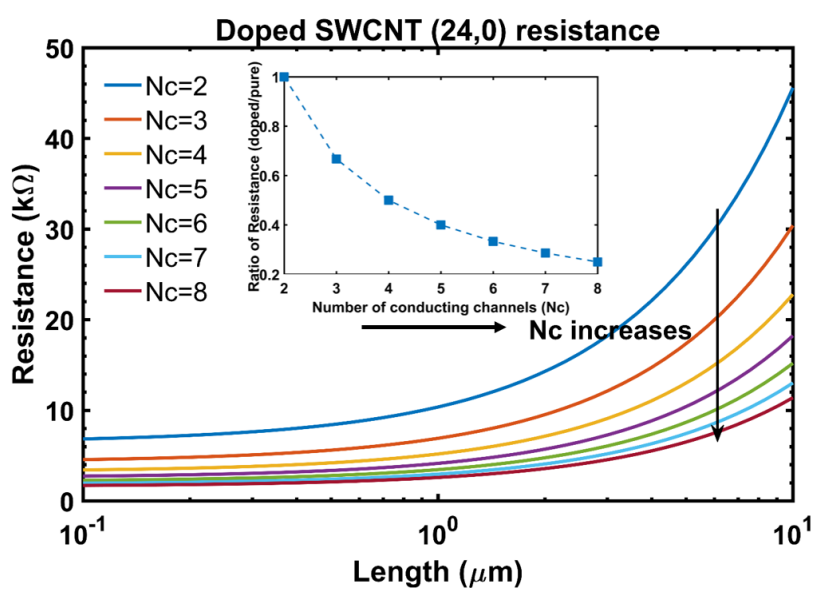

Fig. 6. Resistance of doped SWCNT $(24,0)$ varying with length with different numbers of conducting channels $N_{C}$. inset: resistance ration after increasing $N_{C}$ (doping level).

resistance is reduced up to $80 \%$ (see Fig. 6 inset). Having a fixed diameter over different lengths, we note that doping can reduce the overall resistance. In this plot, we do not consider the defects and contact resistance.

Figure 7 shows the delay ratio of a pristine SWCNT with and without defects over different lengths. Contact resistance is set to 0 . This plot is derived based on the values of defective resistance from Table 1 . We can see the maximum delay ratio happens on SWCNT length around $15 \mu \mathrm{m}$ regardless of the number of defects. Delay ratio increases before $L=15 \mu \mathrm{m}$, but stays steady or decreases when SWCNT lengths get longer. To understand this phenomenon, we note from equation (1), the total SWCNT resistance depends on scattering resistance (first part where $L_{m f p}$ is included) and defective resistance with same impacts on delay. For shorter length, the delay is strongly influenced by defective resistance, while scattering resistance gets more dominant when SWCNT length is much longer than the electron mean free path $L_{m f p}$, where the delay ratio remains steady or decreasing.

In Fig. 8, we compare a doped $(24,0)$ SWCNT $(L=1$ $\mu \mathrm{m})$ with defects to a pristine SWCNT. No contact resis- 


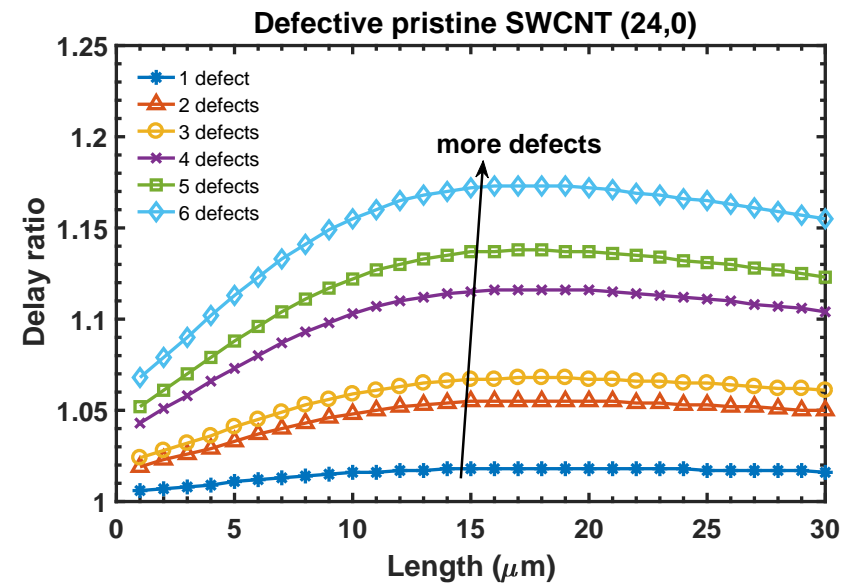

Fig. 7. Delay ratio of pristine SWCNT $(24,0)$ with and without defects over different length.

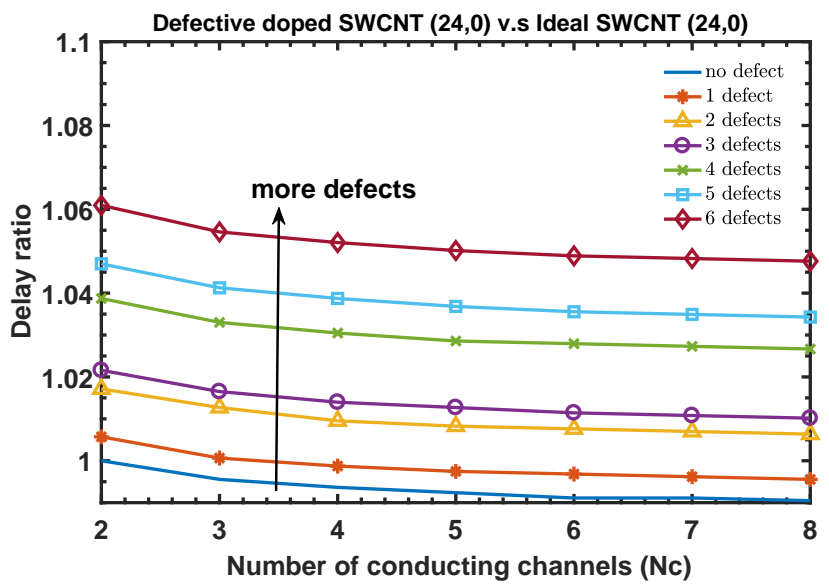

Fig. 8. Delay ratio of defective doped SWCNT vs. pristine ideal SWCNT.

tance is used here. Fig. 8 shows the delay ratio. We know that increasing the doping concentration (i.e., larger $N_{C}$ ) can reduce the resistance, but it will increase the quantum capacitance $C_{Q}$, whereas the electrostatic capacitance $C_{E}$ will remain constant, therefore, the total capacitance remains unchanged. Hence, doping could reduce the propagation delay as shown in the straight blue line where no-defect SWCNT delay ratio decreases with doping concentration. However, additional defective resistance will increase the delay. We can see $6 \%$ of delay is increased with 6 defects, but by introducing doping $\left(N_{C}=8\right)$, delay ratio is reduced by $1 \%$. We also note that occurrence of a single (1) defect with doping of $\left(N_{C}=\right.$ $3)$ can remain the delay ratio equal to 1 .

Figure 9 shows the delay ratio between doped $(24,0)$ SWCNT with contact resistance and pristine SWCNT. In these plots, we do not take into account the defects. Fig. 9 (a) shows the delay ratio for SWCNT length $L=1 \mu \mathrm{m}$ and we observe some trade-off phenomena. If delay ratio keeps inferior to 1 , we can tolerate up to $4 \mathrm{k} \Omega$ of contact resistance with doping of $N_{C} \geq 3$; whereas we need doping to compensate a contact resistance of $8 \mathrm{k} \Omega$. We observe a few overlapping parts between different configurations, which
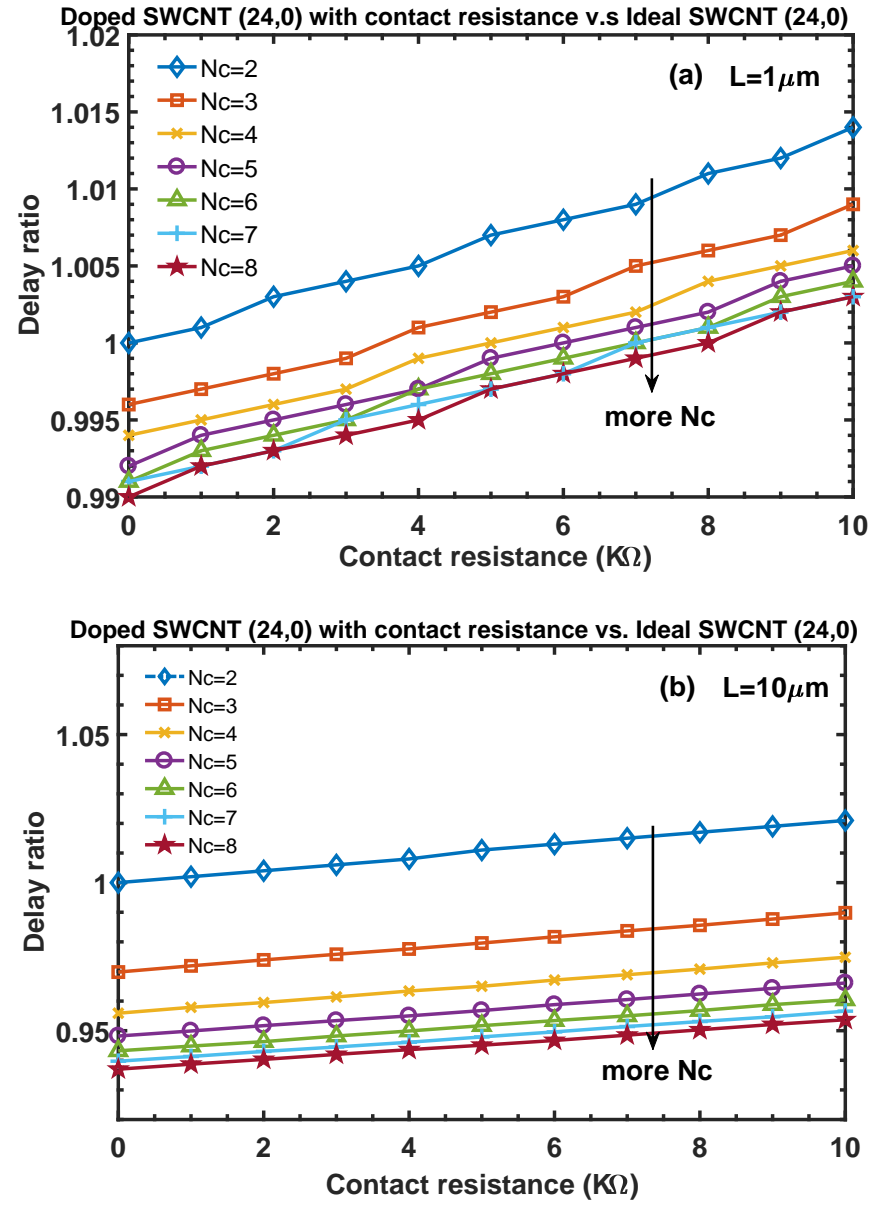

Fig. 9. Delay ratio of doped SWCNT with contact vs. ideal SWCNT without contact. (a) SWCNT length $L=1 \mu \mathrm{m}$. (b) SWCNT length $L=10 \mu \mathrm{m}$.

can be quite interesting for interconnect architecture design with short lengths. Fig. 9 (b) shows the same delay ratio comparison but with a length $L=10 \mu \mathrm{m}$. Overlapping parts are disappearing, but with doping of $N_{C} \geq 3$ a contact resistance of $10 \mathrm{k} \Omega$ can be tolerated to keep the delay ratio under 1 . Comparing to Fig. 9 (a), doping has much stronger impacts on delay for longer SWCNT. With contact resistance as great as $10 \mathrm{k} \Omega$ and doping of $N_{C}=8$, short SWCNT has a delay ratio slightly larger than 1 , whereas $5 \%$ of delay reduction is observed for longer SWCNT.

Overall, we observe that large diameter SWCNTs $(\geq 5 \mathrm{~nm})$ will be more suitable for on-chip interconnects thanks to its lower resistance. Moreover, doping can be applied to reduce further SWCNT resistance. Presence of vacancy (i.e., monoor di-vacancies) defects induces a large defective resistance, especially on short SWCNTs. Poor contacts which exhibit a large contact resistance worsen SWCNT interconnect performance. Charge-based doping helps to mitigate some of the impacts of defects and poor contacts. However, as resistance due to defects and poor contacts increases, doping becomes less efficient. 


\section{CONCLUSION}

A hierarchical model, from atomistic- to circuit-level, has been developed and investigated. We study the defective resistance and number of conducting channels by doping for a metallic zigzag $(24,0)$ SWCNT by using TB-NEGF and DFTGGA on atomistic simulations. We investigate circuitlevel electrical compact modeling and simulations to evaluate the SWCNT interconnect propagation delay with multiple dependencies (defects, doping, contacts). We observed up to $17 \%$ of delay can be increased by defects. However, $80 \%$ of SWCNT resistance can be reduced by doping as well as more than $10 \%$ of defective SWCNT delay is mitigated by doping. Some trade-offs are also observed between the impact of defects, contact resistance and doping on interconnect performance, which can be of interest for circuit level interconnect architecture design. By introducing doping and carefully choosing SWCNT lengths, interconnect performance can be improved while alleviating defects and contact resistances.

\section{ACKNOWLEDGMENT}

Authors would like to thank H2020 CONNECT European project. This project has received funding from the European Unions Horizon 2020 research and innovation program under grant agreement No 688612. (http://www.connect-h2020.eu/)

\section{REFERENCES}

[1] W. Steinhögl, G. Schindler, G. Steinlesberger, M. Traving, and M. Engelhardt, "Comprehensive study of the resistivity of copper wires with lateral dimensions of $100 \mathrm{~nm}$ and smaller," Journal of Applied Physics, vol. 97, no. 2, p. 023706, 2005.

[2] A. Pyzyna, H. Tsai, M. Lofaro, L. Gignac, H. Miyazoe, R. Bruce, C. Breslin, M. Brink, D. Klaus, M. Guillorn et al., "Resistivity of copper interconnects at $28 \mathrm{~nm}$ pitch and copper cross-sectional area below 100 $\mathrm{nm}$ 2," IEEE International Interconnect Technology Conference (IITC), pp. 1-3, 2017.

[3] Itrs 2.0. [Online]. Available: http://www.itrs2.net.

[4] A. Karkar, T. Mak, K.-F. Tong, and A. Yakovlev, "A survey of emerging interconnects for on-chip efficient multicast and broadcast in manycores," IEEE Circuits and Systems Magazine, vol. 16, no. 1, pp. 58-72, 2016.

[5] A. Todri-Sanial, J. Dijon, and A. Maffucci, Carbon Nanotubes for Interconnects. Springer, 2017 ISBN: 978-3-319-29744-6.

[6] C. Subramaniam, T. Yamada, K. Kobashi, A. Sekiguchi, D. N. Futaba, M. Yumura, and K. Hata, "One hundred fold increase in current carrying capacity in a carbon nanotube-copper composite," Nature communications, vol. 4, no. 2202, 2013.

[7] A. R. Harutyunyan, G. Chen, T. M. Paronyan, E. M. Pigos, O. A. Kuznetsov, K. Hewaparakrama, S. M. Kim, D. Zakharov, E. A. Stach, and G. U. Sumanasekera, "Preferential growth of single-walled carbon nanotubes with metallic conductivity," Science, vol. 326, no. 5949, pp. 116-120, 2009.

[8] K. K. Koziol, C. Ducati, and A. H. Windle, "Carbon nanotubes with catalyst controlled chiral angle," Chemistry of Materials, vol. 22, no. 17, pp. 4904-4911, 2010.

[9] S. Esconjauregui, L. D’Arsié, Y. Guo, J. Yang, H. Sugime, S. Caneva, C. Cepek, and J. Robertson, "Efficient transfer doping of carbon nanotube forests by moo3," ACS nano, vol. 9, no. 10, pp. 10422-10430, 2015.

[10] J. Liang, R. Ramos, J. Dijon, H. Okuno, D. Kalita, D. Renaud, J. Lee, V. P. Georgiev, S. Berrada, T. Sadi, A. Asenov, B. Uhlig, K. Lilienthal, A. Dhavamani, F. Könemann, B. Gotsmann, G. Goncalves, B. Chen, K. Teo, R. R. Pandey, and A. Todri-Sanial, "A Physics-Based Investigation of Pt-Salt Doped Carbon Nanotubes for Local Interconnects," IEEE International Electron Devices Meeting (IEDM), pp. 35-5, 2017.

[11] M. Bockrath, W. Liang, D. Bozovic, J. H. Hafner, C. M. Lieber, M. Tinkham, and H. Park, "Resonant electron scattering by defects in single-walled carbon nanotubes," Science, vol. 291, no. 5502, pp. 283285, 2001.
[12] "Atomistix toolkit version 2016.4, quantumwise a/s (www.quantumwise.com)."

[13] E. Aghabararian and A. Shahhoseini, "Effect of relative rotation of walls on the conductance of double-walled carbon nanotubes," IEEE Electrical Engineering (ICEE), 23rd Iranian Conference, pp. 1063-1066, 2015.

[14] J. Lee, S. Berrada, J. Liang, T. Sadi, V. P. Georgiev, A. Todri-Sanial, D. Kalita, R. Ramos, H. Okuno, J. Dijon, and A. Asenov, "The impact of vacancy defects on cnt interconnects: From statistical atomistic study to circuit simulations," IEEE Simulation of Semiconductor Processes and Devices (SISPAD) Conference, pp. 157-160, 2017.

[15] N. Srivastava and K. Banerjee, "Performance analysis of carbon nanotube interconnects for vlsi applications," IEEE/ACM International conference on Computer-aided design, pp. 383-390, 2005.

[16] H. Li, W.-Y. Yin, K. Banerjee, and J.-F. Mao, "Circuit modeling and performance analysis of multi-walled carbon nanotube interconnects," IEEE Transactions on electron devices, vol. 55, no. 6, pp. 1328-1337, 2008.

[17] J. Liang, L. Zhang, N. Azemard-Crestani, P. Nouet, and A. TodriSanial, "Physical description and analysis of doped carbon nanotube interconnects," IEEE International Workshop on Power and Timing Modeling, Optimization and Simulations (PATMOS), pp. 250-255, 2016.

[18] J. Jiang, J. Dong, H. Yang, and D. Xing, "Universal expression for localization length in metallic carbon nanotubes," Physical Review B, vol. 64, no. 4, p. 045409, 2001.

[19] A. D. Franklin and Z. Chen, "Length scaling of carbon nanotube transistors," Nature nanotechnology, vol. 5, no. 12, p. 858, 2010.

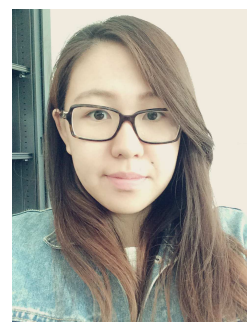

Jie Liang Jie Liang received the B.S. degree in Physics from Paris Denis-Diderot (Paris 7) University, Paris, France in 2014 and the M.S. degree in Quantum Devices from Paris Denis-Diderot (Paris 7) University, Paris, France in 2016. She is currently a $\mathrm{PhD}$ student at the University of Montpellier, France. She is working on the European H2020 project of CNT interconnects.

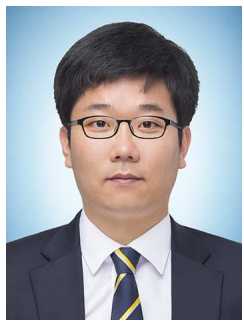

Jaehyun Lee Jaehyun Lee received the B.S., M.S., and Ph.D. degrees in electrical engineering from the Korea Advanced Institute of Science and Technology, Daejeon, South Korea, in 2006, 2008, and 2016, respectively. From 2008 to 2012, he was involved in low power DRAM development with SK Hynix, Icheon, South Korea. He is currently a Research Associate with the School of Engineering, University of Glasgow.

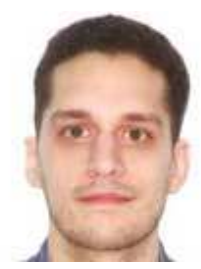

Salim Berrada Salim Berrada received a M.S. in Micro and Nanotechnologies and a Ph.D. degree in Physics both from Paris-Sud University in 2010 and 2014 respectively. He also served as an associated professor in INSA Euro-Mditerrane in Fès, Morocco during the academic year 2015-2016. He is currently a Research Associate with the School of Engineering, University of Glasgow. 


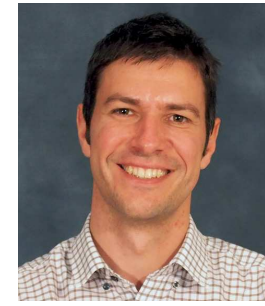

Vihar P. Georgiev Vihar P. Georgiev - (M12) received his Ph.D. degree in computational chemistry from University of Oxford, Oxford, U.K., in 2011. He was an Associate Post-Doctoral Researcher in Device Modelling Group, University of Glasgow, Glasgow, U.K., from 2012 to 2015. Currently he is a Lecturer at University of Glasgow and a deputy group of Device Modelling Group at University of Glasgow. His research includes device modelling, molecular electronics, quantum chemistry and quantum technology.

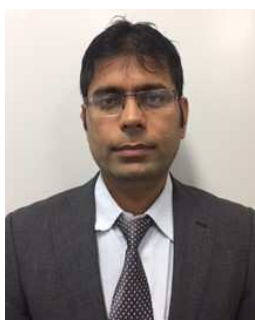

Reeturaj Pandey Dr. Reetu Raj Pandey joined CNRS-LIRMM/University of Montpellier, France as a postdoc researcher in June 2017. He received his Ph.D. from Kyushu Institute of Technology, Japan in April 2017. Dr. Pandey has worked as junior specialist in University of California, Merced USA in 2015 on a short-term project. He received his M.tech degree in Microelectronics from Department of Electronics, Indian Institute of Technology in 2012 and B.Tech degree in Electronics and Communication engineering in 2009. His research interests include micro-nano device fabrications, Graphene and Carbon Nanotube based devices, sensors, semiconductor electronics.

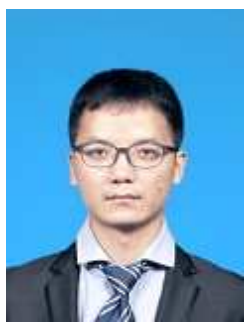

Rongmei Chen Rongmei Chen received the B.S. and Ph.D. degrees in Engineering Physics and Nuclear Technology in 2012 and 2017 respectively from Tsinghua University, Beijing, China. He got the second prize for his Ph.D. thesis of Tsinghua University. He is currently working as a Post-doc in CNRS-LIRMM, Montpellier, France in the field of carbon nanotube based microelectronics.

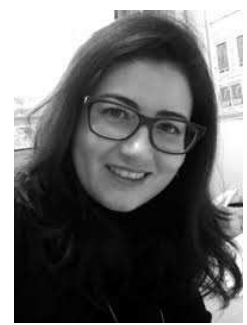

Aida Todir-Sanial Aida Todri-Sanial received her $\mathrm{Ph} . \mathrm{D}$. degree in Electrical and Computer Engineering from the University of California Santa Barbara, USA in 2009. She is currently a Research Scientist at CNRS (French National Center for Scientific Research) and member of the Microelectronics Department at LIRMM since October 2011. Prior to joining CNRS, she was an R\&D Engineer at Fermilab, IL, USA. She has also several visiting research position at Cadence Design Systems, CA, USA (2002-2004), Mentor Graphics, CA, USA (2005), IBM TJ Watson Research Center, NY, USA (2006-2007), STMicroelectronics, Grenoble, France (2008). Dr. Todri-Sanial is recipient of several awards, ACM Distinguished Speaker, CNRS Medaille de Bronze 2016, CNRS Scientific Excellence Award (PES) 2012, John Bardeen Fellow in Engineering at Fermilab 2009, Best Teaching Assistant at Univ. California Santa Barbara, 2006, etc. She has published over 100 papers in VLSI design area.

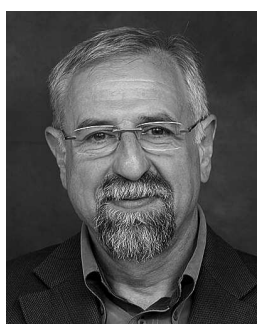

Asen Asenov Asen Asenov (M96-SM05-F11) received the PhD degree in Solid State Physics from the Bulgarian Academy of Science, Sofia, Bulgaria, in 1989. He is the James Watt professor of Electrical Engineering at University of Glasgow and a former CEO of Gold Standard Simulations, Ltd, now part of Synopsys. 